\title{
High Performance Multicast Routing Protocol in Mobile Ad-Hoc Network
}

\author{
Selvendran.R \\ Research Scholar, \\ Department of CSE, \\ B.S. Abdur Rahman University \\ Vandalur, Chennai, India.
}

\author{
Sharmila Sankar, PhD \\ Professor and Head, \\ Department of CSE, \\ B.S. Abdur Rahman University \\ Vandalur, Chennai, India.
}

\author{
M. Sandhya, PhD \\ Professor, \\ Department of CSE, \\ B.S. Abdur Rahman University \\ Vandalur, Chennai, India.
}

\begin{abstract}
This paper intends to improve the performance of MANETS Mobile Ad-hoc networks handling high volume traffic by introducing an enhanced tree based multicast routing protocol with efficient management of topology and groups with an improvised label mechanism capable of building alternate paths and secure better transmission stability. A comparative analysis of the simulation results have shown that the proposed protocol is better performing than the existing multicast routing protocols with slightly higher control overhead.
\end{abstract}

\section{General Terms}

Mobile Ad-hoc Networks( MANETS)

\section{Keywords}

Multicast, Routing Protocol, Topology.

\section{INTRODUCTION}

We ask that authors follow some simple guidelines. In essence, we ask you to make your paper look exactly like this document. The easiest way to do this is simply to download the template, and replace the content with your own material With the growing usage of MANETS (Mobile Adhoc networks) in the recent years in various fields of application like military operations, environmental surveillance, health care and management of commercial activities etc.., handling higher rate of transmissions, improving the performance of the same is an implied necessity. With the advent of mass usage of mobile devices in the daily activities of people involved in various fields, up gradation of existing protocols used in the present day scenario with high performing ones has become an inevitable phenomenon.

Enhancing the performance of multicast routing is a key aspect to improve the overall performance of MANETS with increasing number of nodes in the network. Multicast routing in MANETS is achieved by the following to methods.

Flooding: A mechanism wherein a message recipient node floods the same to its neighbours listed in a table

Routing Tables: Data is transmitted from source node to the destination node via the path constructed in the routing table.

On Demand Paths: Upon receiving a request from a destination node, the source node builds a path from source to destination and then transmits the data via the built path to the destination after getting response from the destination node.

The mechanism of multicast routing can be MESH-BASED or TREE-BASED. In mesh-based multicast routing, more than one transmission path is built and maintained thereby ensuring better data delivery rate and path stability which prevents data loss. However, the mesh-based routing suffers from managing the problem of control overhead since considerable bandwidth is consumed by the control packets used by the mechanism to maintain the topology and routes. On the other hand, the treebased routing while considerably reducing the control overhead by using a core node to manage the group is ineffective in the data delivery front since it could not ensure path stability as it it has no mechanism in hand to repair failed paths and build alternate paths thereby leading to data loss during transmission.

Considering the above points, it is inferred that Mesh based routing can be effective only for MANETS of small scale with less number of groups. In a practical scenario with MANETS involving higher transmission flows, maintaining control packets for the growing number of nodes to ensure path stability will have a deterring effect in data delivery by increasing delay and will also consume considerable bandwidth with control packets. Therefore, tree-based routing coupled with better path stability is considered to be the need for MANETS handling high volume data transmissions. The proposed of protocol is designed to achieve both the aspects of effective data delivery and efficient management of path stability by building alternate paths with comparatively low control overhead.

\section{RELATED WORK}

In recent years, multicast routing attracts extensive research and development activities, the two types of multicast routing protocols are Shortest Path Tree (SPT) based multicast routing protocols and Shared Tree(ST) based multicast routing protocols. For each pair i.e., (Source and Group) rooted at the source SPT builds a separate tree for it. The Distance Vector Multicast Routing (DVMRP)[1] and MOSPF[2] Multicast Extensions to Open Shortest Path First Protocol are SPT based Protocols. In DVMRP, the multicast tree is built by "flooding and pruning". For a specified group if the source router has multicast packets, it floods the packets throughout the network. A router which does not belong to group sends back a prune message. When the process of pruning flooding gets completed, the SPT multicast tree is constructed to connect the source router to the destination router using the shortest delay path. When the topology changes or if a group member joins or leaves a group Distance Vector Multicast Routing DVMRP relies on the membership joining information and the updated periodical information in order to maintains the dynamic multicast tree topology. Multicast Extensions to Open Shortest Path First Protocol (MOSPF) makes use of the feature of the Open Shortest Path First Protocol (OSPF)[3] where each router keeps the topology of the network link state information to construct the Shortest 
Path Tree (SPT) multicast tree. By adding a new type of packet Multicast Extensions to Open Shortest Path First Protocol (MOSPF) extends Open Shortest Path First Protocol (OSPF). This new type of packet is the group membership Link State Advertisement (LSA) which helps I pinpointing the location of all group members. The information about a host joining and leaving the group is distributed by flooding the Link State Advertisement (LSA) packet throughout the network. An identical multicast tree is build for each pair(source, group) based on the information of the network topology and the group membership Link State Advertisement (LSA) packet. A single tree for the entire group is created by Shared Tree(ST) based protocols, which is Shared by all sources. With the help of some mechanism, the Shared Tree(ST)is me a root an a core route and that is publicized. The Shared Tree(ST) based protocols are Core-Based Tree(CBT)[4], Protocol-independent Multicast Sparse Mode(PIM-SM)[5], and Simple Multicast(SM)[6]. In CoreBased Tree(CBT), each group has a corresponding core, using some election mechanism or hash function this router is chosen. The multicast tree is rooted at the core, which is shared by all the sources. The multicast tree is construction the below procedure a join message is send by the host that needs to join the group using the shortest path to the core once it is reaches the core the join message stops it is already on the tree now acknowledgement message is send by the core or the router or the core to the post joining host and the core becomes the part of the tree once it receives the acknowledgement. Once the entire tree is established, a source transfer the packets, the first packet is sending to the core, and then to the group of members.

The PIM-SM protocol is quite similar to CBT, but it also allows for creating a source based shortest path tree on behalf of their attached group members. Thus, MANETS are expected to handle more number of multicast groups and hence any protocol designed for MANETS should be able to handle higher network loads and perform optimally in harsher environments enduring the high volume of nodes and data traffic.

\section{THE PROPOSED PROTOCOL}

This paper discusses the features of two existing multicast routing protocols namely EODMRP (mesh-based) and POEM (Tree-based) so as to compare their performance with the proposed protocol which uses tree-based multicast routing coupled with the ability to build alternate paths using an improved labeling mechanism. In EODMRP, the source node periodically floods the network with control packets to identify and maintain the nodes in the group. However, with increasing number of nodes or recipients in the network, the number of control packets also increases thereby leading to higher consumption of bandwidth which in turn increases the delivery delay time and reduces success ratio in data transmission.

On the other hand, POEM uses the tree-based method of labeling the nodes to build and manage the topology wherein each node is assigned a unique label to identify them during path building. However, POEM fails in the data delivery front due to absence of alternate paths when path failures occur which is quiet phenomenal in a highly dynamic MANET where nodes keep on changing their locations.

The proposed protocol tries to address the path failure occurring in tree based routing by building alternate paths from source to destination unlike the typical tree based protocols. To achieve this it uses labels and a conditional alternate path transmission mechanism with relatively low control overhead in comparison with mesh based routing protocols. By building alternate paths, it effectively manages path failure by providing better path stability and considerably improves data delivery rate by reducing the control packets at a reasonably good cost.

The workflow of the protocol is as follows:

\subsection{Topology Building}

A core node is assigned to broadcast control packets to all the nodes in the topology. Each node that receives the control packet for the first time is labeled and additional labels are kept in reserve at the buffer for dynamic labelling.

\subsection{Data Transmission}

Upon receiving a request from one of the nodes, the source node using the label of the requesting node builds the path from the source to the destination and then transmits the data. The source node will also check for the condition whether building an alternate path is necessary to process the request by checking all the destination nodes for similar labels. If no similar labels are available, the data is transmitted through the single built path. If similar labels are available towards the destination, the source node uses the alternate paths to transmit data if the original path gets failed thus achieving effective transmission success ratio with improved efficiency in path management.

\section{DISCUSSION OF RESULTS}

With the two existing protocols on NS-2 and simulation we have proposed our new multicast routing protocol called as High Performance Multicast Routing Protocol (HPMRP). A $1000 \mathrm{~m} * 1000 \mathrm{~m}$ area with 150 nodes is experimentally set up. The performance is compared after evaluating the HPMRP, EODMRP and POEM. The speed of the node vary from 1 to $10 \mathrm{~m} / \mathrm{s}$, the pause time of each node have mean $30 \mathrm{~ms}$. The duration of simulation is done at least 20 times; the results are normalized to remove topologies because of node placement. The control packet overhead, packet delivery ratios, data endto-end delay are the performance measures of interest.

The total number of groups vs the result of the overhead is explained in figure 1. the assumption of the network topology is static, the multicast group is changed from 1 to 5 , the member in group is divided by a factor. Consider a group network having 10 nodes; it's our priority to increase the 2 group network members to 20. (For example one group can have 13 members and the other can have 7). Thus the figure 2 which gives the delivery ratios vs total number of groups. EODMRP and POEM performs well when the number of groups is set to 2. And EODMRP yields better performance than that of POEM because of the topology, if the number of the multicast group is set to 3 . When the number increases over 3 amount of control packets have to be increased abruptly in EODMRP, and EODMRP depends on the source and it has to maintain the multicast groups and the entire topology. When the number of multicast groups increases it is capable of producing excessive control packets.

Thus, the network may get overloaded which cause additional delay time which in turn degrades the packet delivery ratios as the figure shows. Both the HPMRP and POEM protocols are tree based using label mechanisms to maintain the groups and topologies. 


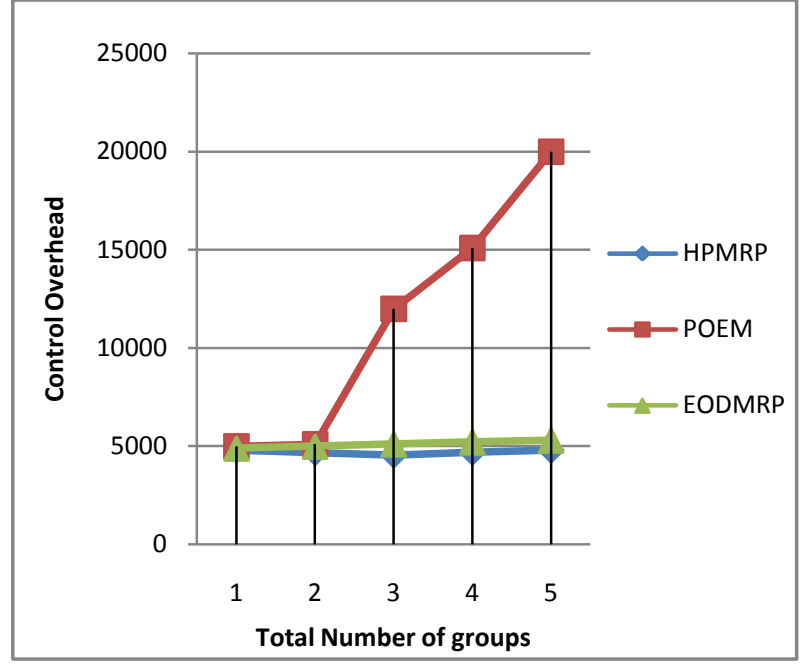

Fig 1: Control overhead vs. Total number of groups.

As the multicast groups grow, our protocol takes more control packets. Other than maintaining the topology and the multicast groups, to facilitate data transmission the label mechanism should create the backup routing paths. Finally, when the multicast group rise to 5 , the performance of our protocol will be $80 \%$ delivery ratios i.e. $15 \%$ more than EODMRP and $10 \%$ more than POEM.

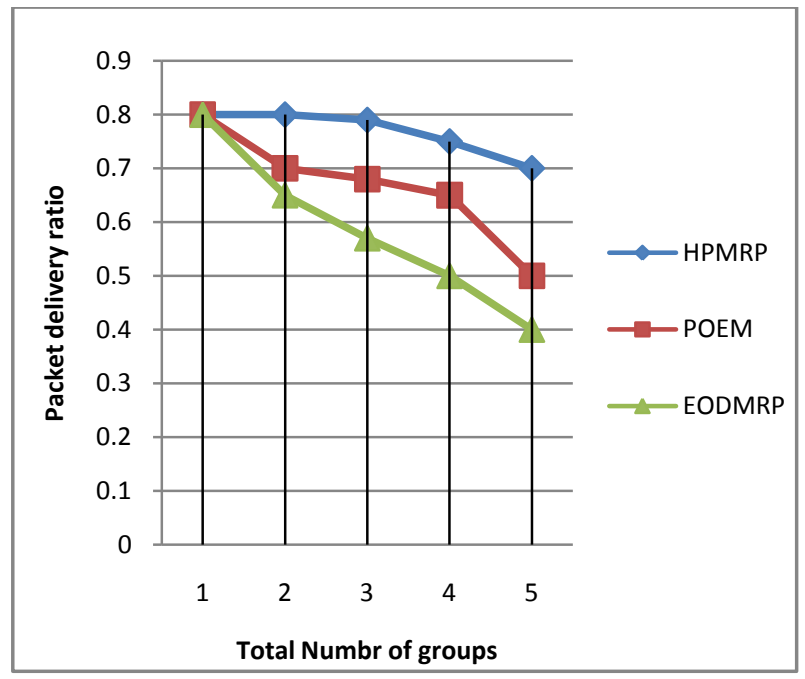

Fig 1: Packet delivery ratio vs. Total number of groups.

Even under harsh environments through many simulations, amidst many multicast groups in the network which brings heavier network loads or even in unexpected situations our protocol constantly produces better performance. The central design or the beck up mechanism is our matter of interest which yields good performance helps us to work under heavy network loads. More control packets may be consumed but the cost is worth when it is compared with the performance gain in path stability, delay time and delivery ratios.

In real time scenario, MANETS are expected to handle more number of multicast groups and hence any protocol designed for MANETS should be able to handle higher network loads and perform optimally in harsher environments enduring the high volume of nodes and data traffic. The simulation test results show that the proposed protocol of ours shows better performance in both the data delivery front as well as tackling path failure with alternate path mechanism with a relatively low control overhead.

\section{CONCLUSIONS}

The protocol proposed in this paper suits well for MANETS with high transmission flow since the design uses the simple tree-based mechanism of building and managing the network topology and group. Further, the tree-based design incorporates the alternate path mechanism of mesh-based routing effectively by an advanced labeling mechanism with relatively low control overhead. With alternate paths in place, the protocol considerably increases the path stability in the network. With relatively low control packets, it consumes lower bandwidth thereby reducing data delivery delay time and effectively improving the transmission success ratio.

\section{REFERENCES}

[1] Waitzman D., and Partridge C., 1988. Distance Vector Multicast Routing Protocol. RFC 1075.

[2] Moy J. 1998. Multicast Extension to OSPF, Internet draft, work in progress.

[3] Moy J., 1998. OSPF Version 2, RFC 2328.

[4] Ballardie, A., Cain B., and Zhang Z. 1998. Core Based Trees (CBT Version 3) Multicast Routing, Internet draft, work in progress.

[5] Deering S. et al. 1998. Protocol Independent MulticastSparse Mode (PIM-SM): Motivation and Architecture, Internet draft, work in progress.

[6] Perlman R. et al. 1999. Simple Multicast: A Design for Simple, Low- Overhead Multicast, Internet draft, work in progress.

[7] Zhiwei Yan, Jong-Hyouk Lee, Sean Shen, and Chunming Qiao. 2013. Novel Branching-Router-Based Multicast Routing Protocol with Mobility Support, IEEE Transactions On Parallel And Distributed Systems.

[8] Luo Junhai, Ye Danxia, Xus Liu, and Fan Mingyu, 2009. A Survey of Multicast Routing Protocol for Mobile AdHoc Networks, IEEE COMMUNICATIONS SURVEYS \& TUTORIALS, VOL. 11, NO. 1, FIRST QUARTER

[9] Sreenath N., Amuthan A., Selvigirija P., 2012. Countermeasures against Multicast attacks on EnhancedOn Demand Multicast Routing Protocol in MANETs, IEEE Computer Communication and Informatics (ICCCI).

[10] Kumar D.R., Najjar W.A., and Srimani P.K., 2001. A New Adaptive Hardware Tree-Based Multicast Routing in K-Ary N-Cubes", IEEE Trans. Computers.

[11] Ji L. and Corson M. S., 1998. A lightweight adaptive multicast algorithm, Proc. IEEE GLOBECO.

[12] Garcia-Luna-Aceves J. J., Madruga E. L., 1999. Coreassisted mesh protocol, IEEE J. Select. Areas Communication.

[13] Guokai Zeng, Bo Wang,Yong Ding, Li Xiao Matt W. Mutka, 2010. Efficient Multicast Algorithms for Multichannel Wireless Mesh Networks", IEEE TRANSACTIONS ON PARALLEL AND DISTRIBUTED SYSTEMS.

[14] Laxman H., Sahasrabuddhe, Biswanath Mukherjee, 2000. Multicast Routing Algorithms and Protocols:A Tlutorial, IEEE Network. 
[15] Chen-Hsiang Feng, Yuqun Zhang, Ilker Demirkol, Wendi Heinzelman B. 2012. Stateless Multicast Protocol for Ad Hoc Networks, IEEE Transactions On Mobile Computing.

[16] Xiang X., Wang X., Yang Y., 2011. Supporting Efficient and Scalable Multicasting over Mobile Ad Hoc Networks , IEEE Transactions On Mobile Computing.

[17] Mohammad-Amin Kharraz, HamidSarbazi-Azad, AlbertY.Zomaya, 2012. On-demand multicast routing protocol with efficient route discovery, Elsevier Journal of Network and Computer Applications.

[18] Le The Dung, Beongku An 2012. A Location-Based Algorithm for Supporting Multicast Routing in Mobile Ad-Hoc Networks , Springer Journal of Network and Parallel Computing,

[19] Yousefi S. and Maleki M.,2013. Multicast scheduling algorithm supporting spatial mini-slot reuse for IEEE 802.16 mesh networks, IEEE Journal on selected areas in communications. 